Original research article

\title{
Predicting the conversion of mild cognitive impairment to Alzheimer's disease based on the volumetric measurements of the selected brain structures in magnetic resonance imaging
}

\author{
Marta Nesteruk ${ }^{a, *}$, Tomasz Nesteruk $^{b}$, Maria Styczyńska ${ }^{c}$, Anna Barczak ${ }^{c}$, \\ Monika Mandecka ${ }^{c}$, Jerzy Walecki ${ }^{d}$, Maria Barcikowska-Kotowicz ${ }^{a, c}$ \\ ${ }^{a}$ Department of Neurology, Central Clinical Hospital of the Ministry of Interior, Warsaw, Poland \\ ${ }^{\mathrm{b}}$ Department of Radiology, Central Clinical Hospital of the Ministry of Interior, Warsaw, Poland \\ ${ }^{\mathrm{c}}$ Department of Neurodegenerative Disorders, Mossakowski Medical Research Centre, Polish Academy of Sciences, \\ Warsaw, Poland \\ ${ }^{\mathrm{d}}$ Mossakowski Medical Research Centre, Polish Academy of Sciences, Warsaw, Poland
}

\section{A R T I C L E I N F O}

Article history:

Received 25 June 2015

Accepted 4 September 2015

Available online 15 September 2015

Keywords:

Mild cognitive impairment

Conversion

Alzheimer's disease

Volumetry

Magnetic resonance imaging

\section{A B S T R A C T}

Introduction: Mild cognitive impairment (MCI) is defined as abnormal cognitive state, but does not meet the criteria for the diagnosis of dementia. According to the new guidelines Alzheimer's disease (AD) involves not only dementia's phase but also predementia phase which is asymptomatic and pathological process in the brain is already present. For this reason it is very important to determine the suitability of markers which should be positive before onset of the first symptoms. One of these biomarkers is a structural magnetic resonance imaging with hippocampal volumetric assessment.

The aim of this study was to investigate the usefulness of structural brain magnetic resonance imaging with volumetric assessment of the hippocampus and entorhinal cortex, posterior cingulate gyrus, parahippocampal gyrus, temporal gyri: superior, medial and inferior, to predict the conversion of $\mathrm{MCI}$ to $\mathrm{AD}$.

Material and methods: Magnetic resonance imaging of brain was performed at the baseline visit in 101 patients diagnosed with MCI. Clinic follow-ups were scheduled after 6.12 and 24 months. Results: Amongst 101 patients with MCI, 17 (16.8\%) converted into AD within two years of observation. All measured volumes were lower in converters than non-converters. Discriminant analysis was conducted and sensitivity for MCI conversion to AD was $64.7 \%$, specificity $96.4 \% .91 \%$ of patients were correctly classified (converter or non-converter). Conclusions: Volumetric measurements may help clinicians to predict MCI conversion to AD but due to low sensitivity it cannot be use separately. The study group requires further observation.

(C) 2015 Polish Neurological Society. Published by Elsevier Sp. z o.o. All rights reserved.

* Corresponding author at: Neurology Clinic, Central Hospital of the Ministry of Interior in Warsaw, 137 Woloska Str., 02-507 Warsaw, Poland. Tel.: +48 2250818 60; fax: +48 225081880 .

E-mail address: msuchcicka@gmail.com (M. Nesteruk).

http://dx.doi.org/10.1016/j.pjnns.2015.09.003

0028-3843/ (C) 2015 Polish Neurological Society. Published by Elsevier Sp. z o.o. All rights reserved. 


\section{Introduction}

Mild cognitive impairment (MCI) is defined as abnormal cognitive state, but does not meet the criteria for the diagnosis of dementia. In the past MCI was treated as a transitional state between the physiological aging and dementia. Currently it is a separate diagnosis, although it is considered that MCI is a stage of pathophysiological process of AD [1]. Patients with MCI do not have problems with function in daily life, sometimes they only need minimal aid [2]. Patient diagnosed with MCI can present stable, non-progressive symptoms until the end of his life, but there can also be observed progression of the disease (usually gradual), leading to the development of dementia. It is estimated that the percentage of the conversion to Alzheimer's disease (AD) is about 10-15\% per year [3]. Criteria for diagnosis of MCI from 2011 [1] in addition to core clinical criteria distinguish research criteria. The research criteria include an assessment of the biological markers of neurons' injury, namely:

- markers of $\beta$-amyloid $(A \beta)$ deposition by assessing the levels of $A \beta 42$ in cerebrospinal fluid or PET amyloid imaging

- markers of neuronal injury by evaluating the levels of total and phosphorylated tau protein or a reduction in hippocampal volume, or atrophy of the medial temporal lobe in the magnetic resonance imaging, or hypometabolism in FDGPET, or hypoperfusion in SPECT.

These criteria are important because pathophysiological process of $\mathrm{AD}$ can last even twenty years before onset of clinical symptoms and MCI is considered as a stage of this pathophysiological processes following the preclinical phase of Alzheimer's disease. In MCI phase biomarkers should be already positive (both markers of $A \beta$ deposition and markers of neuronal injury). A sensitive biomarker will play key role when causative treatment of $\mathrm{AD}$ will be found because then it will be possible to treat this disease even before the onset of clinical symptoms.

The aim of this study was to investigate the usefulness of structural brain magnetic resonance imaging with volumetric assessment of the hippocampus and entorhinal cortex, posterior cingulate gyrus, parahippocampal gyrus, temporal gyri: superior, medial and inferior, to predict the conversion of MCI to AD.

The first abnormalities in patients who are at risk of AD are positive markers of deposition of $\beta$-amyloid (decreased $A \beta$ concentration in the cerebrospinal fluid and/or positive PET amyloid imaging). In this study the marker of neuronal injury was used - structural MRI with volumetric assessment of selected brain structures (inter alia hippocampus, entorhinal cortex) because of good availability of magnetic resonance in Poland (the best of neuroimaging biomarkers) and its noninvasive nature. Furthermore, according to Jack, in the MRI pathological changes are observed as one of the last biomarkers [4] and our patients were not in the preclinical phase - they were diagnosed with MCI. Reduction of the measured volumes was expected in patients who were at risk of conversion to AD.

\section{Material and methods}

In the study initially participated 163 patients diagnosed with MCI who were evaluated in Alzheimer's Department of MSW Hospital in Warsaw. 101 patients remained for further data analysis (due to lack of follow-up visits or errors in MRI which precluded further analysis of the data). The patients were aged 50-80 years, mean age 62.7. Neurological assessment, MMSE (Mini-Mental State Examination), CDT (clock drawing test) and GDS (Global Deterioration Scale) were performed. The average score of MMSE was 27.4/30 (range 25-30 points). The average score of CDT was $8.5 / 10$ points (range $6-10$ points) and the mean score of GDS 2.7 points (range 2-3 points). Laboratory tests included morphology, TSH, blood glucose, urea, creatinine, transaminases, VDRL (veneral disease research laboratory), levels of vitamin B12 and folic acid and there was no significant abnormalities. Each patient was assessed using standard neuropsychological tests (according to the standards described by Gabryelewicz [5]). On CDR scale (Clinical Dementia Rating), all patients received 0.5 or $0 / 0.5$. For each patient, follow-up visits were scheduled after 6 months ( \pm 14 days), 12 months ( \pm 30 days) and 24 months ( \pm 50 days). Then patients were again evaluated neurologically (included MMSE, CDT, GDS assessment). Neuropsychological examination was performed to assess potential disease progression. In patients diagnosed with conversion to $\mathrm{AD}$ acetylcholinesterase inhibitor treatment was initiated. All patients remain under the care of the Memory Disorders Outpatient Clinic of MSW Hospital in Warsaw.

Brain MRI was performed for each of the patients on a $1.5 \mathrm{~T}$ Toshiba apparatus in the Department of Radiology of MSW Hospital. Scans were obtained in T2-weighted, FLAIR, DWI images and also thin 3D $\mathrm{T} 1$ Alzheimer sequence. Using FreeSurfer software volumes of selected structures were calculated, that is: hippocampus, entorhinal cortex, posterior cingulate gyrus, parahippocampal gyrus, temporal gyri: superior, medial, inferior and total intracranial volume. Subsequently, the results have been checked by the same radiologist to detect and eliminate possible errors which may arise in the process of segmentation. Each volume (hippocampus, entorhinal cortex, posterior cingulate gyrus, parahippocampal gyrus, temporal gyri: superior, medial, inferior) was divided by the total intracranial volume to normalize results and to make possible comparison between patients and eliminate differences in the brain size (according to Whitwell [6]). All volumes were multiplied by 1000 in order to facilitate comparison between them.

\section{Results}

17 of the 101 patients diagnosed with MCI converted into AD within two years of observation, namely $16.8 \%$ (distribution of diagnosed patients for each visit, Table 1). The study population was divided into two subgroups:

- Subgroup 1 - non-converters, who did not converted into AD (84 patients).

- Subgroup 2 - converters, who converted into AD (17 patients). 
Table 1 - Distribution of diagnosed patients for each visit.

\begin{tabular}{lrccc} 
& Visit 1 & $\begin{array}{c}\text { Visit after } \\
6 \text { months }\end{array}$ & $\begin{array}{c}\text { Visit after } \\
12 \text { months }\end{array}$ & $\begin{array}{c}\text { Visit after } \\
\text { 24 months }\end{array}$ \\
\hline MCI & 101 & 92 & 85 & 84 \\
$\mathrm{AD}$ & 0 & 9 & 16 & 17 \\
\hline
\end{tabular}

Average decrease of the volumes in converters compared to non-converters was achieved for all volumetric measurements, Table 2. The hypothesis of a normal distribution was positively verified for all measurements (the results of the Kolmogorov-Smirnov test, Table 3). The significance of intergroup differences was obtained for the majority of measurements except: left posterior cingulate gyrus $(t=1.659, p=0.100)$, left superior temporal gyrus $(t=1.274, p=0.206)$ and right posterior cingulate gyrus $(t=1.202, p=0.232)$. The most significant differences between groups were found for: left hippocampus $(t=5.708, p<0.001)$, left entorhinal cortex $(t=5.473$, $p<0.001)$, right hippocampus $(\mathrm{t}=5.129, p<0.001)$, right inferior temporal gyrus $(t=3.803, p<0.001)$ and right entorhinal cortex $(t=3.676, p<0.001)$, Table 3 .

Discriminant analysis was conducted and sensitivity for MCI conversion to AD was $64.7 \%$, specificity $96.4 \%$. $91 \%$ of patients were correctly classified (converter or non-converter). Discriminant analysis was also conducted in the modified version with an automatic selection of variables which had the highest discrimination power. The final model took into account only two variables, which provide the best group membership: the volume of the left hippocampus and left entorhinal cortex. Discriminant analysis model using these

\section{Table 2 - Descriptive statistics in each subgroup (normalized volumes were multiplied by 1000).}

\begin{tabular}{|c|c|c|c|c|c|c|}
\hline & \multicolumn{2}{|c|}{ Nonconverters $(n=84)$} & \multicolumn{2}{|c|}{ Converters $(n=17)$} & \multicolumn{2}{|c|}{ All $(n=101)$} \\
\hline & Average & $\begin{array}{l}\text { Standard } \\
\text { deviation }\end{array}$ & Average & $\begin{array}{l}\text { Standard } \\
\text { deviation }\end{array}$ & Average & $\begin{array}{l}\text { Standard } \\
\text { deviation }\end{array}$ \\
\hline Left hippocampus & 2.489 & 0.333 & 1.971 & 0.381 & 2.402 & 0.392 \\
\hline Right hippocampus & 2.493 & 0.366 & 1.998 & 0.348 & 2.410 & 0.406 \\
\hline Left entorhinal cortex & 0.593 & 0.114 & 0.426 & 0.120 & 0.565 & 0.131 \\
\hline Right entorhinal cortex & 0.490 & 0.104 & 0.389 & 0.099 & 0.473 & 0.110 \\
\hline Left posterior cingulate gyrus & 1.649 & 0.239 & 1.543 & 0.237 & 1.631 & 0.241 \\
\hline Right posterior cingulate gyrus & 1.615 & 0.319 & 1.516 & 0.246 & 1.598 & 0.309 \\
\hline Left parahippocampal gyrus & 1.145 & 0.181 & 0.969 & 0.247 & 1.115 & 0.203 \\
\hline Right parahippocampal gyrus & 1.035 & 0.164 & 0.902 & 0.223 & 1.012 & 0.181 \\
\hline Left inferior temporal gyrus & 5.961 & 0.777 & 5.092 & 1.061 & 5.815 & 0.888 \\
\hline Left medial temporal gyrus & 5.449 & 0.699 & 4.999 & 0.844 & 5.373 & 0.740 \\
\hline Left inferior temporal gyrus & 6.043 & 0.796 & 5.754 & 1.101 & 5.994 & 0.855 \\
\hline Right inferior temporal gyrus & 5.806 & 0.843 & 4.926 & 0.997 & 5.658 & 0.927 \\
\hline Right medial temporal gyrus & 6.236 & 0.816 & 5.735 & 0.844 & 6.151 & 0.838 \\
\hline Right inferior temporal gyrus & 6.021 & 0.789 & 5.518 & 1.053 & 5.936 & 0.854 \\
\hline
\end{tabular}

Table 3 - Results of a Student's t-test.

\begin{tabular}{|c|c|c|c|c|c|c|c|}
\hline & \multirow{2}{*}{$\begin{array}{c}\text { KS normality test } \\
\text { Significance }\end{array}$} & \multicolumn{2}{|c|}{$\begin{array}{l}\text { Levene's homogeneity of } \\
\text { variance }\end{array}$} & \multicolumn{4}{|c|}{$\begin{array}{c}\text { Mean difference for independent samples in a student's } \\
\text { t-test }\end{array}$} \\
\hline & & $F$ & Significance & $\mathrm{T}$ & $\begin{array}{l}\text { Degree of } \\
\text { freedom }\end{array}$ & $\begin{array}{l}\text { Significance } \\
\text { (two-sided) }\end{array}$ & $\begin{array}{l}\text { Average } \\
\text { difference }\end{array}$ \\
\hline LH & 0.265 & 1.254 & 0.266 & 5.708 & 99 & $0.000^{* *}$ & 0.518 \\
\hline LERC & 0.821 & 0.235 & 0.629 & 5.473 & 99 & $0.000^{* *}$ & 0.168 \\
\hline $\mathrm{RH}$ & 0.434 & 0.055 & 0.814 & 5.129 & 99 & $0.000^{* *}$ & 0.495 \\
\hline RITG & 0.416 & 2.659 & 0.106 & 3.803 & 99 & $0.000^{* *}$ & 0.88 \\
\hline RERC & 0.66 & 0.084 & 0.772 & 3.676 & 99 & $0.000^{* *}$ & 0.101 \\
\hline LITG & 0.573 & 4.047 & 0.047 & 3.208 & 19.622 & $0.004^{* *}$ & 0.869 \\
\hline LPHG & 0.846 & 4.45 & 0.037 & 2.783 & 19.605 & $0.012^{*}$ & 0.176 \\
\hline LMTG & 0.738 & 1.257 & 0.265 & 2.338 & 99 & $0.021^{*}$ & 0.45 \\
\hline RPHG & 0.725 & 4.445 & 0.038 & 2.32 & 19.63 & $0.031^{*}$ & 0.132 \\
\hline RMTG & 0.866 & 0.173 & 0.679 & 2.294 & 99 & $0.024^{*}$ & 0.501 \\
\hline RSTG & 0.757 & 3.812 & 0.054 & 2.26 & 99 & $0.026^{*}$ & 0.503 \\
\hline LPCG & 0.767 & 0.038 & 0.847 & 1.659 & 99 & 0.1 & 0.105 \\
\hline LSTG & 0.722 & 3.373 & 0.069 & 1.274 & 99 & 0.206 & 0.289 \\
\hline RPCG & 0.449 & 0.266 & 0.607 & 1.202 & 99 & 0.232 & 0.098 \\
\hline \multicolumn{8}{|c|}{$\begin{array}{l}\text { Abbreviations used in the table: LH - left hippocampus, LERC - left entorhinal cortex, RH - right hippocampus, RITG - right inferior temporal } \\
\text { gyrus, RERC - right entorhinal cortex, LITG - left inferior temporal gyrus, LPHG - left parahippocampal gyrus, LMTG - left medial temporal } \\
\text { gyrus, RPHG - right parahippocampal gyrus, RMTG - right medial temporal gyrus, RSTG - right superior temporal gyrus, LPCG - left posterior } \\
\text { cingulate gyrus, LSTG - left superior temporal gyrus, RPCG - right posterior cingulate gyrus. } \\
p<0,05 \text {. } \\
p<0,01 .\end{array}$} \\
\hline
\end{tabular}


two variables was statistically significant. Sensitivity of the measurements of the left hippocampus and left entorhinal cortex in the prediction of MCI progression to AD was $58.8 \%$. Sensitivity for these measurements in predicting the stable form of MCI was the same as using all the measured parameters, namely $96.4 \%$, and diagnosis classification accuracy using left hippocampus and left entorhinal cortex was 90\% (for all measurements 91\%). The highest sensitivity in predicting $\mathrm{MCI}$ conversion to $\mathrm{AD}$, using a single measurement was for left hippocampus (52.9\%), right hippocampus (41.2\%) and left entorhinal cortex (41.2\%).

\section{Discussion}

In descriptive statistics presented in Table 2, average volumes of all structures are smaller in converters comparing to non-converters (according to the preliminary assumptions). However, statistical analysis showed the superiority of some measurements. The highest sensitivity of conversion for single structure was obtained for the left hippocampus (52.9\%), right hippocampus (41.2\%) and left entorhinal cortex (also 41.2\%). Unsuitable measurements for assessing MCI to $\mathrm{AD}$ conversion were the volumes of: left and right posterior cingulate gyrus, left and right superior temporal gyrus and right medial temporal gyrus (the sensitivity was $0 \%$ ). Our results are consistent with the sequence of neuropathological changes described by Braak [7,8], where entorhinal cortex and hippocampus are affected as the first by the neuropathological process and subsequently cortex of temporal lobes in stage III and IV. Volumes of superior temporal gyri proved to be unsuitable probably because of their main role in the process of auditory sensation, so their role in cognitive processes is significantly lower. The most of the published studies has evaluated usefulness of hippocampus and entorhinal cortex volumes in the prediction of MCI conversion to AD. Our study also confirms the highest value of these two parameters. Studies which assessed additionally, for example, volume of parahippocampal gyrus, indicated that the use of this parameter did not increase prediction sensitivity for conversion to $\mathrm{AD}[9,10]$. In our study the use of all measured volumes increased sensitivity from $58.8 \%$ (where the volume of the left hippocampus and left entorhinal cortex was used) to $64.7 \%$. Specificity in both cases was $96.4 \%$. Significant differences between the sensitivity and specificity figures resulted from subgroup size difference ( 17 converters and 84 with stable MCI). In other studies, the percentage of conversion to $\mathrm{AD}$ was higher, namely $27-45 \%$ [9-11]. In the few studies [10,12,13] the most sensitive volumetric measurement was entorhinal cortex volume. In our study left entorhinal cortex volume was less sensitive measurement in comparison with left hippocampus volume, as in the Ewers' work [14]. Patients with MCI are a heterogeneous group and may be at different stages of neuropathological process, that is why use of hippocampus and entorhinal cortex volumes seems be the most optimal method. Volumetric measurements of hippocampus, entorhinal cortex, posterior cingulate gyrus, parahippocampal gyrus and temporal gyri: superior, medial, inferior showed a relatively low sensitivity in predicting the conversion of $\mathrm{MCI}$ to $\mathrm{AD}$ (in the analysis of all measurements $64.7 \%$ ). Higher sensitivity was obtained for predicting stable MCI (96.4\%).

The limitation of our study was the number of patients (101). Studies which drew from either ADNI database (the Alzheimer's Disease Neuroimaging Initiative) or AddNeuroMed program present the results from significantly larger groups of patients. For example Risacher et al. [15] analyzed 339 patients with MCI from ADNI database. 18.3\% converted into AD per year. Bilateral hippocampal volumes, entorhinal cortex and temporal gyri thickness were significantly reduced in MCI-converters in comparison to the MCI-stable participants which is consistent with our results except for the left superior temporal gyrus (LSTG), Table 3. In our study the volume of LSTG was not significantly lower, but in Risarcher study the thickness, not volume of temporal gyri was measured. Another study [16] based on ADNI database (Li et al.) covered 293 patients with $\mathrm{MCI}$ and the observation period lasted 4 years. To predict conversion from MCI to AD the authors used not only the measured volumes but also demographic, genetic information, cognitive scores, and the results of laboratory tests. They employed a hierarchical interaction model to select the most predictive biosignatures and interactions between all of the parameters to perform correct classification the patients with MCI (converter or nonconverter). The accuracy of classification was almost $75 \%$ (the most effective MRI parameters were precuneus, amygdala, entorhinal cortex and hippocampus). Percentage of the correct classification in our study using all volumes was $91 \%$ but sensitivity was only $64.7 \%$. Higher correct classification rate in our study was probably due to the large differences between subgroups (17 converters vs. 84 non-converters). In Li's study conversion rate was 45\% (132 converters vs. 161 nonconverters), so subgroups were almost equivalent.

The study from 2014 [17] presents results using data from both mentioned databases (ADNI and AddNeuroMed) and involved 345 patients with MCI. The authors used ordinal regression model to 1023 baseline structural MRI scans. They modeled all classes simultaneously (in study were also AD patients and control group) and applied multivariate ordinal regression from which they extract a probabilistic prediction of class membership and an index of AD progression - the ORCHID score. Classification accuracy was 70-75\% (ADNIAddNeuroMed); sensitivity of $77 \%$ and specificity of $72 \%$.

This study confirmed results from 2011 [18] in which multivariate classification methods for MRI was used. Data was from AddNeuroMed database. MCI group was 101 patients (like in our study) and 19 patients converted into AD during one year observation. The authors compared correct classification accuracy between visual assessment rating scales, a multivariate MRI classification method (with using regional volume and regional cortical thickness measures, providing 57 variables for multivariate analysis) and manually measured hippocampal volumes. The best accuracy for predicting conversion was for the model with automated regional MRI measures $-79 \%$ and $68 \%$ accuracy was obtained to other two methods. Comparable group size with our study proved that using sophisticated statistical tools can improve the efficiency of the method.

Our study group requires further observation, because in the following years the proportion of the patients who 
progressed to $\mathrm{AD}$ compared to patients remaining in a stable condition may change.

\section{Conflict of interest}

None declared.

\section{Ethics}

The work described in this article has been carried out in accordance with The Code of Ethics of the World Medical Association (Declaration of Helsinki) for experiments involving humans; Uniform Requirements for manuscripts submitted to Biomedical journals.

\section{Acknowledgement and financial support}

None declared.

\section{R E F E R E N C E S}

[1] Albert MS, DeKosky ST, Dickson D, Dubois B, Feldman HH, Fox NC, et al. The diagnosis of mild cognitive impairment due to Alzheimer's disease: recommendations from the National Institute on Aging-Alzheimer's Association workgroups on diagnostic guidelines for Alzheimer's disease. Alzheimers Dement 2011;7:270-9.

[2] Winblad B, Palmer K, Kivipelto M, Jelic V, Fratiglioni L, Wahlund LO, et al. Mild cognitive impairment - beyond controversies, towards a consensus: report of the International Working Group on Mild Cognitive Impairment. J Intern Med 2004;256:240-6.

[3] Petersen RC, Roberts RO, Knopman DS, Boeve BF, Geda YE, Ivnik RJ, et al. Mild cognitive impairment: ten years later. Arch Neurol 2009;66(December (12)):1447-55.

[4] Jack Jr CR, Albert MS, Knopman DS, McKhann GM, Sperling RA, Carrillo MC, et al. Introduction to the recommendations from the National Institute on Aging-Alzheimer's Association workgroups on diagnostic guidelines for Alzheimer's disease. Alzheimers Dement 2011;7:257-62.

[5] Gabryelewicz T, Styczynska M, Luczywek E, Barczak A, Pfeffer A, Androsiuk W, et al. The rate of conversion of mild cognitive impairment to dementia: predictive role of depression. Int J Geriatr Psychiatry 2007;22(6):563-7.
[6] Whitwell JL, Crum WR, Watt HC, Fox NC. Normalization of cerebral volumes by use of intracranial volume: implications for longitudinal quantitative MR imaging. AJNR Am J Neuroradiol 2001;22(September (8)):1483-9.

[7] Braak H, Braak E. Evolution of the neuropathology of Alzheimer's disease. Acta Neurol Scand Suppl 1996;165:3-12.

[8] Braak H, Braak E. Staging of Alzheimer's disease-related neurofibrillary changes. Neurobiol Aging 1995;16:271-8.

[9] Convit A, de Asis J, de Leon MJ, Tarshish CY, De Santi S, Rusinek H. Atrophy of the medial occipitotemporal, inferior, and middle temporal gyri in non-demented elderly predict decline to Alzheimer's disease. Neurobiol Aging 2000;21 (January-February (1)):19-26.

[10] Devanand DP, Pradhaban G, Liu X, Khandji A, De Santi S, Segal S, et al. Hippocampal and entorhinal atrophy in mild cognitive impairment prediction of Alzheimer disease. Neurology 2007;68:828-36.

[11] Pennanen C, Kivipelto M, Tuomainen S, Hartikainen P, Hänninen T, Laakso MP, et al. Hippocampus and entorhinal cortex in mild cognitive impairment and early AD. Neurobiol Aging 2004;25(March (3)):303-10.

[12] Dickerson BC, Goncharova I, Sullivan MP, Forchetti C, Wilson RS, Bennett DA, et al. Aging MRI-derived entorhinal and hippocampal atrophy in incipient and very mild Alzheimer's disease. Neurobiol 2001;22(September-October (5)): :747-54.

[13] Stoub TR, Bulgakova M, Leurgans S, Bennett DA, Fleischman D, Turner DA, et al. MRI predictors of risk of incident Alzheimer disease: a longitudinal study. Neurology 2005;64(May (9)):1520-4.

[14] Ewers M, Walsh C, Trojanowski JQ, Shaw LM, Petersen RC, Jack Jr CR, et al. North American Alzheimer's Disease Neuroimaging Initiative (ADNI) Prediction of conversion from mild cognitive impairment to Alzheimer's disease dementia based upon biomarkers and neuropsychological test performance. Neurobiol Aging 2012;33(July (7)):1203-14.

[15] Risacher SL, Saykin AJ, West JD, Shen L, Firpi HA, McDonald $\mathrm{BC}$, et al. Baseline MRI predictors of conversion from MCI to probable AD in the ADNI cohort. Curr Alzheimer Res 2009;6 (August (4)):347-61.

[16] Li H, Liu Y, Gong P, Zhang C, Ye J, Alzheimers Disease Neuroimaging Initiative. Hierarchical interactions model for predicting Mild Cognitive Impairment (MCI) to Alzheimer's Disease (AD) conversion. PLoS ONE 2014;9 (January (1)):e82450. http://dx.doi.org/10.1371/journal. pone.0082450

[17] Doyle OM, Westman E, Marquand AF, Mecocci P, Vellas B, Tsolaki M, et al. Predicting progression of Alzheimer's disease using ordinal regression. PLoS ONE 2014;9(August (8)):e105542. http://dx.doi.org/10.1371/journal.pone.0105542

[18] Westman E, Cavallin L, Muehlboeck JS, Zhang Y, Mecocci P, Vellas B, et al. Sensitivity and specificity of medial temporal lobe visual ratings and multivariate regional MRI classification in Alzheimer's disease. PLoS ONE 2011;6(7):e22506. 